\title{
Isoparametric hypersurfaces in a Randers sphere of constant flag curvature
}

\author{
Ming $\mathrm{Xu}^{1}$
}

Received: 30 April 2017 / Accepted: 30 September 2017 / Published online: 12 October 2017

(C) Fondazione Annali di Matematica Pura ed Applicata and Springer-Verlag GmbH Germany 2017

\begin{abstract}
In this paper, I study the isoparametric hypersurfaces in a Randers sphere $\left(S^{n}, F\right)$ of constant flag curvature, with the navigation datum $(h, W)$. I prove that an isoparametric hypersurface $M$ for the standard round sphere $\left(S^{n}, h\right)$ which is tangent to $W$ remains isoparametric for $\left(S^{n}, F\right)$ after the navigation process. This observation provides a special class of isoparametric hypersurfaces in $\left(S^{n}, F\right)$, which can be equivalently described as the regular level sets of isoparametric functions $f$ satisfying $-f$ is transnormal. I provide a classification for these special isoparametric hypersurfaces $M$, together with their ambient metric $F$ on $S^{n}$, except the case that $M$ is of the OT-FKM type with the multiplicities $\left(m_{1}, m_{2}\right)=(8,7)$. I also give a complete classification for all homogeneous hypersurfaces in $\left(S^{n}, F\right)$. They all belong to these special isoparametric hypersurfaces. Because of the extra $W$, the number of distinct principal curvature can only be 1,2 or 4 , i.e. there are less homogeneous hypersurfaces for $\left(S^{n}, F\right)$ than those for $\left(S^{n}, h\right)$.
\end{abstract}

Keywords Randers sphere of constant flag curvature · Isoparametric function · Isoparametric hypersurface $\cdot$ Homogeneous hypersurface $\cdot$ Navigation

Mathematics Subject Classification $53 \mathrm{C} 60 \cdot 53 \mathrm{C} 42 \cdot 22 \mathrm{E} 46$

\section{Introduction}

In Riemannian geometry, the study on isoparametric hypersurfaces has a long history. It is defined as a regular level set for an isoparametric function $f$ on a Riemannian manifold $\left(N, d s^{2}\right)$, i.e.

Supported by NSFC (no. 11771331).

Ming Xu

mgmgmgxu@163.com

1 School of Mathematical Sciences, Capital Normal University, Beijing 100048,

People's Republic of China 


$$
|\nabla f|^{2}=a(f), \quad \text { and } \quad \Delta f=b(f),
$$

in which $a(\cdot)$ is smooth and $b(\cdot)$ is continuous.

The classification of isoparametric hypersurfaces in space forms is a classical geometric problem with a history of almost one hundred years. Those in Euclidean and hyperbolic spaces were classified in 1930's $[6,28,30]$. But for the most difficult case, those in a unit sphere, the classification work occupied a long list of works [7-11,13,14,18,20-22,24,25,32], and was recently completely solved [12]. Much more references can be found in the good survey paper [31]. Isoparametric functions and isoparametric hypersurfaces on other Riemannian manifolds, especially the exotic spheres, were studied by Tang and his students [27].

On the other hand, isoparametric function and isoparametric hypersurface in Finsler geometry have not been studied until recently He et al. purposed their definitions for a Finsler space [16], satisfying similar conditions as in (1.1). But now the gradient and Laplacian are only smoothly defined on the open set where $d f \neq 0$. Generally speaking, they are nonlinear and hard for calculation, because of the changing base vectors. Most of their good properties in Riemannian geometry cannot be easily generalized.

Studying and classifying isoparametric hypersurfaces in Finsler space forms, i.e. complete simply connected Finsler spaces with constant flag curvature, are interesting problems naturally generalized from Riemannian geometry. But in Finsler geometry, the metrics for space forms can be very complicated, like the examples Bryant constructed on spheres [5]. We know very few about them except some special cases.

In [16] and [17], the authors considered two special cases of Finsler space forms for the ambient space, i.e. Minkowski spaces (with zero flag curvature) and Funk spaces (with negative constant flag curvature). They classified the isoparametric hypersurfaces in them. However, for ambient space with positive constant flag curvature, their progress is relatively slow.

In this paper, I will consider the (non-Riemannian) Randers sphere of constant flag curvature for the ambient space and study a special class of isoparametric hypersurfaces in them, including all the homogeneous ones. Until now, they are the only known examples in Finsler space forms of positive flag curvature. We guess they are the only ones when the ambient space is a Randers sphere of constant flag curvature.

To introduce the special isoparametric hypersurfaces studied in this paper, we need to use the navigation process and the celebrated works of Bao et al. [3] on Randers spheres of constant flag curvature. Briefly speaking, a Randers sphere $\left(S^{n}, F\right)$ of constant flag curvature $\kappa$ is produced by the navigation process with the datum $(h, W)$, such that $\left(S^{n}, h\right)$ is a standard Riemannian sphere with the same constant curvature $\kappa$ (thus we must have $\kappa>0$ ), and $W$ is a Killing vector field for $\left(S^{n}, h\right)$.

The isoparametric hypersurfaces I have studied in this paper are those tangent to the Killing vector field $W$ in the navigation datum $(h, W)$ for the ambient Randers sphere $\left(S^{n}, F\right)$ of constant flag curvature. We see a correspondence between these special isoparametric hypersurfaces of $\left(S^{n}, F\right)$, and those of $\left(S^{n}, h\right)$ which are tangent to $W$. We summarize it as the following theorem.

Theorem 1.1 Let $\left(S^{n}, F\right)$ be a Randers sphere of constant flag curvature 1, corresponding to the navigation datum $(h, W)$. Then for any isoparametric hypersurface $M$ of the unit sphere $S^{n}(1)=\left(S^{n}, h\right)$, we can find a Killing vector field $W$ tangent to $M$, such that $M$ is isoparametric for the Randers sphere $\left(S^{n}, F\right)$ defined by the navigation datum $(h, W)$. Conversely, any isoparametric hypersurface $M$ of $\left(S^{n}, F\right)$ which is tangent to $W$ is isoparametric for the unit sphere $S^{n}(1)$. 
Theorem 1.1 is a summarization of Theorems 4.5, 5.4 and 6.1. In the later three theorems, I have proved something more.

In Theorem 4.5, I have given equivalent descriptions for those special isoparametric hypersurfaces, i.e. they are the regular level sets of isoparametric functions $f$ of a Randers sphere of constant flag curvature, such that $-f$ is also transnormal or isoparametric.

In Theorem 5.4, I point out that any connected homogeneous hypersurface $M$ of a Randers sphere $\left(S^{n}, F\right)$ of constant flag curvature, with respect to the connected isometry subgroup $K=I_{o}\left(S^{n}, M, F\right)$ of $\left(S^{n}, F\right)$ preserving $M$, belongs to the special isoparametric hypersurfaces in Theorems 1.1 or 4.5, i.e. $M$ is tangent to the Killing vector field $W$ in the navigation datum for $F$. I provide a complete classification for these homogeneous hypersurfaces. Notice that, because of the extra $W$ in the metric datum, there are less than the ones in the Riemannian context. Especially, those with the number of distinct principal curvatures $g=3$ or 6 do not appear. Using the theory for CK-vector fields [34,36], I determine all the choices of $W$ for $M$ to be $K$-homogeneous.

In Theorem 6.1, I have calculated the group $G=I_{o}\left(S^{2 l-1}, M, h\right)$ and its Lie algebra for each isoparametric hypersurface of the OT-FKM type in the unit sphere $S^{2 l-1}(1)=$ $\left(S^{2 l-1}, h\right)$, with the multiplicities $m_{1}=m \leq l-m-1=m_{2}$.

The isoparametric hypersurfaces in unit spheres of the OT-FKM type were found and studied in $[14,24,25]$. They can be explicitly constructed using real representations for symmetric Clifford algebras. See Sect. 6 for its definition. It has been known for a long time that most isoparametric hypersurfaces of the OT-FKM type are non-homogeneous, but much recently that they provides all the non-homogeneous isoparametric hypersurfaces in unit spheres $[9,12,18]$.

Theorems 5.4 and 6.1 together determine all the possible choices of $W$ in the navigation datum $(h, W)$ and classify the corresponding ambient metrics for the special isoparametric hypersurfaces in Theorem 1.1, except the case when $\left(m_{1}, m_{2}\right)=(8,7)$ (see the remarks after Theorem 6.1).

This paper is organized as follows. In Sect. 2, I briefly review the fundamental knowledge of Finsler geometry which is needed in later discussion. In Sect. 3, I introduce the isoparametric function and isoparametric hypersurface in Riemannian geometry and Finsler geometry. In Sect. 4, I study the special isoparametric hypersurfaces in Randers spheres of constant flag curvature, which are tangent to the vector field in the navigation datum. In Sects. 5 and 6, I continue to explore them from the view points of homogeneity and Clifford system.

\section{Preliminaries}

In this section, I first briefly summarize some fundamental concepts in Finsler geometry from [1] and [29].

\subsection{Minkowski norm and Finsler metric}

A Minkowski norm on a real vector space $\mathbf{V}, \operatorname{dim} \mathbf{V}=n$, is a continuous function $F: \mathbf{V} \rightarrow$ $[0,+\infty)$ satisfying the following conditions [1]:

(1) $F$ is a positive smooth function on $\mathbf{V} \backslash 0$.

(2) $F(\lambda y)=\lambda F(y)$ for any $y \in \mathbf{V}$ and $\lambda \geq 0$. 
(3) With respect to any linear coordinates $y=y^{i} e_{i} \in \mathbf{V}$, the Hessian matrix

$$
g_{i j}(y)=\left(\frac{\partial^{2}}{\partial y^{i} \partial y^{j}} F^{2}(y)\right)
$$

is positive definite for any $y \neq 0$.

We also call $g_{i j}(y)$ the fundamental tensor. The inverse matrix for $\left(g_{i j}(y)\right)$ is denoted as $\left(g^{i j}(y)\right)$. They are used in Finsler geometry to move indices up or down. Each Hessian matrix $\left(g_{i j}(y)\right)$ with $y \neq 0$ defines an inner product $g_{y}^{F}=\langle\cdot, \cdot\rangle_{y}^{F}$ as following,

$$
\langle u, v\rangle_{y}^{F}=g_{i j}(y) u^{i} v^{j}=\left.\frac{1}{2} \frac{\partial^{2}}{\partial s \partial t} F^{2}(y+s u+t v)\right|_{s=t=0},
$$

for any $u=u^{i} e_{i}$ and $v=v^{i} e_{i}$ in $\mathbf{V}$. It is obvious that the inner product $g_{y}^{F}$ is independent of the linear coordinates.

A Finsler metric on a smooth manifold $M$ is a continuous function $F: T M \rightarrow[0,+\infty)$ which is smooth on the slit tangent bundle $T M \backslash 0$, and its restriction to each tangent space is a Minkowski norm. We also call $(M, F)$ a Finsler manifold or a Finsler space.

For example, Riemannian metrics are Finsler metrics, which Hessian matrices with respect to any standard local coordinates $x=\left(x^{i}\right) \in M$ and $y=y^{i} \partial_{x^{i}} \in T_{x} M, g_{i j}(x, y)$, only depends on $x$. In this case, we usually refer to the smooth section

$$
F^{2}=g_{i j}(x) d x^{i} d x^{j} \in \Gamma\left(\operatorname{Sym}^{2}\left(T^{*} M\right)\right)
$$

as the Riemannian metric. Generally speaking, we will only consider non-Riemannian metrics in Finsler geometry.

Randers metrics are the most simple and important Finsler metrics. They are of the form $F=\alpha+\beta$, where $\alpha$ is a Riemannian metric and $\beta$ is a 1 -form, such that $|\beta(x)|_{\alpha}<1$ at each point $x \in M$. The $(\alpha, \beta)$-metrics are generalizations of Randers metrics, which are of the form $F=\alpha \phi(\beta / \alpha)$ with a positive smooth function $\phi$, and similar $\alpha$ and $\beta$ as for Randers metrics.

There is a canonical way to define a Riemannian metric from a smooth vector field $Y$ on a Finsler space $(M, F)$. Let $\mathcal{U} \subset M$ the open subset where $Y$ is non-vanishing. Then, $Y$ defines a Riemannian metric $g_{Y}^{F}$ on $\mathcal{U}$ by all the Hessian matrices $\left(g_{i j}(x, Y(x))\right)$, i.e. for each standard local coordinates on $\mathcal{U}, g_{Y}^{F}=\sqrt{g_{i j}(x, Y(x)) d x^{i} d x^{j}}$. We call this metric the localization of $F$ at $Y$.

\subsection{Geodesic and flag curvature}

A geodesic on a Finsler space $(M, F)$ is a non-constant smooth curve $c(t): I \rightarrow M$ which satisfies the locally minimizing principle for the arc length functional

$$
L(c(t))=\int_{I} F(c(t), \dot{c}(t)) \mathrm{d} t .
$$

Any geodesic can be re-parametrized such that $F(c(t), \dot{c}(t)) \equiv$ const $>0$. Then it is locally defined by the equations

$$
\ddot{c}^{i}(t)+2 \mathbf{G}^{i}(c(t), \dot{c}(t))=0, \quad \forall i,
$$

where $\mathbf{G}^{i}=\frac{1}{4}\left(\left[F^{2}\right]_{x^{k} y^{l}} y^{k}-\left[F^{2}\right]_{x^{l}}\right)$ is the coefficient of the geodesic spray.

A smooth vector field on $(M, F)$ is a geodesic field, if it is non-vanishing everywhere, and its integration curves are geodesics of $(M, F)$. 
Now we define the flag curvature, which is a generalization for the sectional curvature in Riemannian geometry [29]. Let $y$ be any nonzero tangent vector in $T_{x} M$ and $\mathbf{P}=\operatorname{span}\{y, v\}$ a tangent plane in $T_{x} M$ containing $y$. Then the flag curvature for $(x, y, \mathbf{P})$ is defined by

$$
K^{F}(x, y, \mathbf{P})=K^{F}(x, y, y \wedge v)=\frac{\left\langle R_{y}^{F} v, v\right\rangle_{y}^{F}}{\langle y, y\rangle_{y}^{F}\langle v, v\rangle_{y}^{F}-\left[\langle y, v\rangle_{y}^{F}\right]^{2}},
$$

where $R_{y}^{F}$ is the Riemann curvature, which can be locally presented as $R_{y}^{F}=R_{i}^{k}(y) \partial_{x^{i}} \otimes d x^{k}$ : $T_{x} M \rightarrow T_{x} M$, where

$$
R_{k}^{i}(y)=2 \partial_{x^{k}} \mathbf{G}^{i}-y^{j} \partial_{x^{j} y^{k}}^{2} \mathbf{G}^{i}+2 \mathbf{G}^{j} \partial_{y^{j} y^{k}}^{2} \mathbf{G}^{i}-\partial_{y^{j}} \mathbf{G}^{i} \partial_{y^{k}} \mathbf{G}^{j} .
$$

When $F$ is Riemannian, the flag curvature $K^{F}(x, y, \mathbf{P})$ coincides with the sectional curvature, which only depends on the tangent plane $\mathbf{P} \in T_{x} M$.

\subsection{Navigation process and Randers spheres of constant flag curvature}

The navigation process is an important method for constructing new metrics from an old ones.

Let $F$ be a Finsler metric and $W$ a vector field on $M$ such that $F(W(x))<1$ for each $x \in M$. We denote $\tilde{y}=y+F(x, y) W(x)$ for any $y \in T_{x} M$. Then, the equality $\tilde{F}(\tilde{y})=F(y)$ defines another Finsler metric whose indicatrix $\mathcal{I}_{x}^{\tilde{F}}=\left\{\tilde{y} \in T_{x} M \mid \tilde{F}(x, y)=1\right\}$ is a parallel shifting of the indicatrix $\mathcal{I}_{x}^{F}$ by the vector $W(x)$.

We call $(F, W)$ the navigation datum defining $\tilde{F}$. When $F$ is Riemannian, $\tilde{F}$ is a Randers metric, which can be presented as

$$
\tilde{F}(\cdot)=\frac{1}{\lambda}\left(\sqrt{\lambda F(\cdot)^{2}+\left(\langle W, \cdot\rangle^{F}\right)^{2}}-\langle W, \cdot\rangle^{F}\right),
$$

where $\lambda=1-F(W)^{2}$. All Randers metrics can be produced in this way, and there is a one-to-one correspondence between Randers metrics and navigation data $(h, W)$ in which $h$ is a Riemannian metric.

The navigation process is crucial for studying Randers metrics of constant flag curvature. The following theorem summarized from [3] provides the foundation.

Theorem 2.1 A Randers metric $F$ has the constant flag curvature $\kappa$ if and only if its navigation datum $(h, W)$ satisfies the following conditions:

(1) The metric $h$ has constant curvature $k+\frac{1}{4} \mu^{2}$ for some constant $\mu$.

(2) The vector field $W$ is $\mu$-homothetic for $h$, i.e. $\mathcal{L}_{W} h=2 \mu h$, where $\mathcal{L}$ is the Lie derivative.

Furthermore, for a Randers sphere of constant flag curvature with the navigation datum $(h, W), W$ can only be a Killing vector field, i.e. 0-homothetic for the metric $h$.

Randers spheres of constant flag curvature have many good geometric properties. For example, its $S$-curvature vanishes [2,35]. 


\section{Isoparametric function and isoparametric hypersurface}

In this section, I will recall the definitions of isoparametric function and isoparametric hypersurface in Riemannian geometry and very briefly discuss their classification when the ambient space is a unit sphere. Then, I will introduce their generalization in Finsler geometry.

\subsection{Definitions in Riemannian geometry}

The key feature of an isoparametric function $f$ in Riemannian geometry is that $|\nabla f|$ and $\Delta f$ only depend on values of $f$. Any regular level set of $f$ (i.e. pre-image of regular values of $f$ ) is called an isoparametric hypersurface.

In practice, for some technical reasons (see [33] for example), we use the following definitions. A non-constant smooth function $f$ on a Riemannian manifold $N$ is called transnormal if $|\nabla f|^{2}=a(f)$ for some smooth function $a(\cdot)$. Furthermore a transnormal function $f$ is called isoparametric if $\Delta f=b(f)$ for some continuous function $b(\cdot)$.

For example, if $N$ is simply connected and it admits the cohomogeneity one isometric action of a compact connected Lie group $G$, then we can find a suitable $G$-invariant isoparametric function $f$, such that each principal $G$-orbit is a homogeneous isoparametric hypersurface.

The classification for isoparametric hypersurfaces in unit spheres is one of the most celebrated geometric problems. It has been studied for eighty years and completely solved recently $[9-12,18]$. Briefly speaking, it is either homogeneous or of the OT-FKM type. We will see more detailed descriptions for these two cases in Sects. 5 and 6, respectively.

Any connected imbedded orientable hypersurface in a unit sphere with constant mean curvature is an isoparametric hypersurface. Its principal curvatures, counting multiplicities, are constant functions too, which can be expressed as $\kappa_{i}=\cot \left(\theta+\frac{i-1}{g}\right)$, with multiplicities $m_{i}$, and $m_{i}=m_{i+2}$ where the subindices are $\bmod g$. The only possible $g$ 's are 1,2, 3, 4 and $6[20,21]$.

\subsection{Definitions in Finsler geometry}

Now we define a smooth function $f$ on a Finsler space $(N, F)$ to be isoparametric, which needs proper interpretations for $\nabla f$ and $\Delta f$.

We assume the smooth function $f$ is not constant, i.e. the open subset $\mathcal{U}=\{x \in$ $N \mid d f(x) \neq 0\}$ of $M$ is non-empty. Each level set of $f$ in $\mathcal{U}$ is then a smooth hypersurface. Assume $x \in M=\mathcal{U} \cap f^{-1}(c)$, then there exists a unique vector $\nabla f(x)$ pointing to the increasing direction of $f$ and satisfying

$$
\langle\nabla f(x), v\rangle_{\nabla f(x)}^{F}=\left.d f(v)\right|_{x} .
$$

Then $\nabla f$ defines a smooth vector field on $\mathcal{U}$ which can be continuously extended to $M \backslash \mathcal{U}$ where it equals 0 . We call $\nabla f$ the nonlinear gradient of $f$. It can also be interpreted as the gradient vector field of $f \mid \mathcal{U}$ with respect to the localization metric $g_{\nabla}^{F}$. Notice that generally $-\nabla f \neq \nabla(-f)$.

Similarly, we also use the metric $g_{\nabla f}^{F}$ to define the nonlinear Laplacian $\Delta f$ of $f$, where $\Delta$ is the Laplacian for the Riemannian manifold $\left(\mathcal{U}, g_{\nabla f}^{F}\right)$. Now we are ready to give the following definitions.

Definition 3.1 A non-constant smooth function $f$ on $M$ is called transnormal if $F(\nabla f)$ only depends on values of $f$ when they are restricted to $\mathcal{U}=\{x \in M \mid d f(x) \neq 0\}$. Furthermore, a 
transnormal function $f$ is called isoparametric if $\Delta f$ satisfies the same property on $\mathcal{U}$. Each level set of $f$ in $\mathcal{U}$ is called an isoparametric hypersurface.

The isoparametric condition we propose here is simpler than that in [16]. Two definitions apply the same idea that using the nonlinear gradient $\nabla f$ as the base vector. But their definition for $\Delta f$ involves an arbitrary volume form on $M$, which results an extra summation term $S(\nabla f)$, where $S(\cdot)$ is the $S$-curvature with respect to the chosen volume form [29]. If we choose the $\mathrm{BH}$-volume and discuss the case that the ambient manifold is a Randers sphere of constant flag curvature, there is no difference between the two definitions because the $S$-curvature vanishes.

The transnormal condition can be equivalently presented as

$$
F(\nabla f(x))=a(f(x)), \quad x \in \mathcal{U},
$$

where $a(\cdot)$ is a smooth function on $f(\mathcal{U})$. By Sard theorem $f(\mathcal{U})$ is a dense open set in $f(N)$, and by the transnormal condition $\mathcal{U}=f^{-1}(f(\mathcal{U}))$. But $f$ may have many critical values, which cannot be erased as in Riemannian geometry by adjusting $f$, because $-f$ may not be transnormal.

For example, in an Minkowski space $\left(\mathbb{R}^{n}, F\right)$ with $n>1, S_{F}^{n-1}\left(x_{0}, r\right)=\{x \in$ $\mathbb{R}^{n} \mid d_{F}\left(x_{0}, x\right)=r$ with $r>0$ is an isoparametric hypersurface, corresponding to the isoparametric function $f(x)=d_{F}\left(x_{0}, x\right)$ [16]. If $-f$ is transnormal, by (1) of Lemma 4.1 below, the dual norm $F^{*}$ of $F$ must be reversible, i.e. $F^{*}(y)=F^{*}(-y)$, which implies $F$ is also reversible.

This observation suggests it be an interesting problem to study and classify the special isoparametric hypersurface, whose isoparametric function $f$ is, such that $-f$ is also transnormal (or more strongly, $-f$ is also isoparametric).

\section{Special isoparametric hypersurfaces in Randers spheres of constant flag curvature}

In this section, we study the special isoparametric hypersurfaces proposed at the end of last section, when the ambient manifold is a Randers sphere $\left(S^{n}, F\right)$ of constant flag curvature. We will see they are equivalently characterized by the condition that they are tangent to the Killing vector field $W$ in the navigation datum $(h, W)$ for $F$.

Firstly, we consider a non-constant smooth function $f$ on a Finsler space $(N, F)$ such that $f$ and $-f$ are both transnormal.

There exist positive smooth function $a_{1}(\cdot)$ and $a_{2}(\cdot)$ such that $F(\nabla f)=a_{1}(f)$ and $F(\nabla(-f))=a_{2}(f)$ on $\mathcal{U}=\{x \in N \mid d f(x) \neq 0\}$. Denote

$$
\mathbf{n}_{1}=\frac{\nabla f}{a_{1}(f)} \text { and } \mathbf{n}_{2}=\frac{\nabla(-f)}{a_{2}(f)}
$$

the two unit normal fields along all level sets of $f$ in $\mathcal{U}$. Then, we have the following easy lemma.

Lemma 4.1 (1) The function $f$ defines a Finsler submersion from $(\mathcal{U}, F)$ to the onedimensional manifold $f(\mathcal{U})$, such that the induced Finsler metric $F^{\prime}$ on $f(\mathcal{U})$ satisfy $F^{\prime}\left(f_{*} \mathbf{n}_{1}\right)=F^{\prime}\left(f_{*} \mathbf{n}_{2}\right)=1$.

(2) The vector fields $\mathbf{n}_{1}$ and $\mathbf{n}_{2}$ are geodesic fields on $(\mathcal{U}, F)$ (i.e. their integration curves are geodesics of $(\mathcal{U}, F)$ ). 
See [26] for the theory of Finsler submersion. Here we only use its definition, and the fact that the horizonal lift of a geodesic (field) is a geodesic (field). The proof of the lemma is very easy, so we omit it.

Notice if we only have the transnormal property for $f$, the function $f$ can still be treated as a "submersion for just one side", such that only $\mathbf{n}_{1}$ is a horizonal lift of a geodesic field on $f(\mathcal{U})$. So $\mathbf{n}_{1}$ is geodesic field for $(\mathcal{U}, F)$, but $\mathbf{n}_{2}$ is not in general.

Secondly, we further assume the ambient manifold $(N, F)$ is a Randers space, which corresponds to the navigation datum $(h, W)$.

Let $c$ be the positive smooth function on $\mathcal{U}$ defined by $f_{*} \mathbf{n}_{1}=-c f_{*} \mathbf{n}_{2}$. Then by Lemma 4.1, $c$ only depends on values of $f$. Direct calculation shows $W=\frac{1}{2}\left(\mathbf{n}_{1}+\mathbf{n}_{2}\right)$, and thus $f_{*} W=\frac{1}{2}\left(f_{*} \mathbf{n}_{1}+f_{*} \mathbf{n}_{2}\right)$ is a well defined vector field on $f(\mathcal{U})$.

Thirdly, we further require the ambient space is a Randers sphere $\left(S^{n}, F\right)$ of constant flag curvature, i.e. in its navigation datum $(h, W)$, the Riemannian metric $h$ has a positive constant curvature and $W$ is a Killing vector field for $\left(S^{n}, h\right)$. Then, we claim as follows:

Lemma 4.2 If $f$ is a non-constant smooth function on a Randers sphere of constant flag curvature, such that both $f$ and $-f$ are transnormal, then $f$ is preserved by the flow generated by $W$.

Proof We first observe that $f_{*} W \equiv 0$ in $\mathcal{U}$, i.e. the flow generated by $W$ preserves each regular value of $f$. Assume conversely $f_{*} W \neq 0$ at the pre-image for the regular value $c$ of $f$, then a diffeomorphism generated by $W$ maps $N^{c}=\{x \in N \mid f(x) \leq c\}$ to some other $N^{c^{\prime}}$ with $c^{\prime} \neq c$. It is a contradiction because $W$ is Killing vector field of $(N, h)$, i.e. it generates isometries which preserves the volume defined by $h$.

Because $f(\mathcal{U})$ is a dense open set in $f\left(S^{n}\right)$, by continuity, critical values of $f$ are also preserved by the flow generated by $W$, which ends the proof for the lemma.

Fourthly, we consider the isoparametric condition and prove the following lemma.

Lemma 4.3 If $f$ is an isoparametric function on a Randers sphere $\left(S^{n}, F\right)$ of constant flag curvature, with the navigation datum $(h, W)$, such that both $f$ and $-f$ are transnormal, then $f$ is also isoparametric for the Riemannian metric $h$.

To prove this lemma, we may assume the Killing vector field $W$ is non-vanishing almost everywhere.

When restricted to $\mathcal{U}$, Lemma 4.2 implies that $W$ is tangent to each level set of $f$. Denote $\mathbf{n}=\nabla^{h} f /\left|\nabla^{h} f\right|$ the unit vector field in $\mathcal{U}$, where $\nabla^{h}$ is the gradient operator with respect to $h$. Then $\mathbf{n}_{1}=\mathbf{n}+W, \mathbf{n}_{2}=-\mathbf{n}+W$ and $[\mathbf{n}, W]=0$.

At any point in $\mathcal{U}$ where $W$ does not vanish, we can find local coordinates $x=\left(x^{i}\right)$, $1 \leq i \leq n$, such that $\partial_{x^{1}}=\mathbf{n}, \partial_{x^{2}}=W$ and the level sets of $f$ are given by the equation $x^{1} \equiv$ const. Then, the metric $h$ can be locally presented as

$$
h=\sqrt{\left(d x^{1}\right)^{2}+\sum_{i, j>1} h_{i j} d x^{i} d x^{j}},
$$

where the functions $h_{i j}$ do not depend on $x^{2}$, and $0<h_{22}<1$ (because $W$ is a Killing vector field of $h$ which length at each point is strictly less than 1).

Using above local coordinates, it is obvious to see $f$ is transnormal with respect to $h$. 
To describe the nonlinear Laplacian $\Delta f$, where $\Delta$ is the Laplacian operator with respect to the localization $g_{\nabla f}^{F}=g_{\mathbf{n}_{1}}^{F}$, we define another local coordinate system at $x$ as follows:

$$
\tilde{x}^{2}=x^{2}-x^{1}, \quad \text { and } \tilde{x}^{i}=x^{i} \text { when } i \neq 2 .
$$

Then, $\partial_{\tilde{x}^{1}}=\mathbf{n}_{1}$ and the level sets of $f$ are given by $\tilde{x}^{1} \equiv$ const. So we can similarly present

$$
g_{\mathbf{n}_{1}}^{F}=\sqrt{\left(d \tilde{x}^{1}\right)^{2}+\sum_{i, j>1} g_{i j} d \tilde{x}^{i} d \tilde{x}^{j}}
$$

where $g_{i j}=g_{i j}^{F}\left(x, \mathbf{n}_{1}\right)$. To see the relation between $g_{i j}$ and $h_{i j}$, we need the following lemma.

Lemma 4.4 Let $\tilde{F}$ be the Minkowski norm on $\mathbf{V}$ defined by the navigation datum $(F, v)$, i.e. $\tilde{F}(\tilde{y})=F(y)$ where $\tilde{y}=y+F(y) v$ for each $y \in \mathbf{V}$. Then, for any vector $y$ and $u$ in $\mathbf{V}$ satisfying $y \neq 0$ and $\langle u, y\rangle_{y}^{F}=0$, we have

$$
\langle u, u\rangle_{y}^{F}=\frac{\langle u, u\rangle_{\tilde{y}}^{\tilde{F}}}{1-\langle\tilde{y}, v\rangle_{\tilde{y}}^{\tilde{F}}} .
$$

In particular, $\langle u, u\rangle_{\tilde{y}}^{\tilde{F}}=\langle u, u\rangle_{y}^{F}$ for all $u$ in the $g_{y}^{F}$-complement of $y$ if $\langle v, y\rangle_{y}^{F}=0$.

Proof For any $t$, we have

$$
\tilde{F}^{2}(y+t u+F(y+t u) v)=F^{2}(y+t u) .
$$

Differentiate it twice for the $t$-variable, and take $t=0$, then we get

$$
\langle u, u\rangle_{\tilde{y}}^{\tilde{F}}+\left\langle\tilde{y},\langle u, u\rangle_{y}^{F} \cdot v\right\rangle_{\tilde{y}}^{\tilde{F}}=\langle u, u\rangle_{y}^{F},
$$

and

$$
\langle u, u\rangle_{y}^{F}=\frac{\langle u, u\rangle_{\tilde{y}}^{\tilde{F}}}{1-\langle\tilde{y}, v\rangle_{\tilde{y}}^{\tilde{F}}} .
$$

Because the indicatrix $\mathcal{I}^{\tilde{F}}$ is a parallel shifting of the indicatrix $\mathcal{I}^{F}$, we have $\langle\tilde{y}, v\rangle_{\tilde{y}}^{\tilde{F}}=0$ when $\langle y, v\rangle_{y}^{F}=0$. So we have $\langle u, u\rangle_{\tilde{y}}^{\tilde{F}}=\langle u, u\rangle_{y}^{F}$ in this case.

Notice that in Lemma 4.4, the statuses of $F$ and $\tilde{F}$ are symmetric, i.e. $(\tilde{F},-v)$ is also the navigation datum for $F$. We have $\langle u, \tilde{y}\rangle_{\tilde{y}}^{\tilde{F}}=0$ when $\langle u, y\rangle_{y}^{F}=0$. So (4.3) can also be given as

$$
\langle u, u\rangle_{\tilde{F}}^{\tilde{y}}=\frac{\langle u, u\rangle_{y}^{F}}{1+\langle y, v\rangle_{y}^{F}} .
$$


By Lemma 4.4, we have

$$
\begin{aligned}
g_{i j}\left(\tilde{x}^{1}, \tilde{x}^{2}, \ldots, \tilde{x}^{n}\right) & =h_{i j}\left(x^{1}, x^{2}, \ldots, x^{n}\right) \\
& =h_{i j}\left(\tilde{x}^{1}, \tilde{x}^{2}+\tilde{x}^{1}, \tilde{x}^{3}, \ldots, \tilde{x}^{n}\right) \\
& =h_{i j}\left(\tilde{x}^{1}, \tilde{x}^{2}, \tilde{x}^{3}, \ldots, \tilde{x}^{n}\right),
\end{aligned}
$$

where $i, j>1$.

To summarize, we see that $g_{\mathbf{n}_{1}}^{F}$ has the same local presenting as $h$, except that all $x^{i}$, s are changed to $\tilde{x}^{i}$, s, respectively, and by similar argument, we can prove so does $g_{\mathbf{n}_{2}}^{F}$. All three metrics, $h, g_{\mathbf{n}_{1}}^{F}$ and $g_{\mathbf{n}_{2}}^{F}$ are Riemannian metrics of the same positive constant curvature. By the isoparametric condition, the level sets of $f$ have constant principal curvatures with respect to $g_{\mathbf{n}_{1}}^{F}$; thus, the statement is valid with $g_{\mathbf{n}_{1}}^{F}$ changed to the other two, i.e. $f$ is isoparametric for $\left(S^{n}, h\right)$, and $-f$ is isoparametric for $\left(S^{n}, F\right)$. Notice that by the continuity, this claim is still valid where $W$ vanishes .

Finally, we consider an isoparametric function $f$ for $\left(S^{n}, h\right)$ which is preserved by the flow generated by $W$, where $(h, W)$ is the navigation datum for a Randers sphere $\left(S^{n}, F\right)$ of constant flag curvature. With very minor changes, above argument also proves both $f$ and $-f$ are isoparametric for $\left(S^{n}, F\right)$.

Summarizing above arguments, we have the following theorem.

Theorem 4.5 Let $\left(S^{n}, F\right)$ be a Randers sphere of constant flag curvature, with the navigation datum $(h, W)$. Then, any isoparametric function $f$ of $\left(S^{n}, h\right)$ which is preserved by the flow generated by $W$ is isoparametric for $\left(S^{n}, F\right)$.

Conversely, if $f$ is isoparametric and $-f$ is transnormal for $\left(S^{n}, F\right)$, then $f$ is isoparametric for $\left(S^{n}, h\right)$ and preserved by the flow generated by $W$. Furthermore, $-f$ is also parametric for $\left(S^{n}, F\right)$.

Theorem 4.5 implies that when the ambient space is an Randers sphere of constant flag curvature, the special isoparametric hypersurfaces purposed in the previous section are just those in the classification list in the Riemannian context which are tangent to the vector field $W$ in the navigation datum, or equivalently, preserved by the flow generated by $W$. We will see in the next two sections that each isoparametric hypersurfaces in a unit sphere permits navigation changes for the metric, i.e. Killing vector field tangent to it. Furthermore, all the Killing vector field $W$ tangent to an isoparametric hypersurface for the standard round sphere $\left(S^{n}, h\right)$ (i.e. all the Randers metrics $F$ of constant flag curvature permitting these special isoparametric hypersurfaces) can be determined except the case of the OT-FKM type with multiplicities $\left(m_{1}, m_{2}\right)=(8,7)$.

\section{Homogeneous isoparametric hypersurface}

Assume $\left(S^{n}, F\right)$ is a Randers sphere of constant flag curvature, and $(h, W)$ is its navigation datum. Without loss of generalities, we may assume $\left(S^{n}, h\right)$ is the unit sphere $S^{n}(1)$ and $W$ is a nonzero Killing vector field for it. The connected isometry group $I_{o}\left(S^{n}, F\right)$ of $\left(S^{n}, F\right)$ is the proper subgroup of $I_{o}\left(S^{n}, h\right)=S O(n+1)$ which preserves $W$, or equivalently commutes with $W$ when $W$ is viewed as a matrix in $s o(n+1)$.

Assume $M$ is a connected homogeneous isoparametric hypersurface of $S^{n}(1)=\left(S^{n}, h\right)$. We denote $G=I_{o}\left(S^{n}, M, h\right)$ and $K=I_{o}\left(S^{n}, M, F\right) \subset G$ the maximal connected subgroup of $S O(n+1)$ and $I\left(S^{n}, F\right)$, respectively, preserving $M$. The homogeneity here, in the 
Riemannian context, means that $G$ acts transitively on $M$. All $G$ 's are classified by the following table, which coincides with the isotropy actions of compact rank two symmetric spaces [15].

\begin{tabular}{rllll}
\hline Case & $G=I_{o}\left(S^{n}, M, h\right)$ & $\operatorname{dim} M$ & $g$ & $\left(m_{1}, m_{2}\right)$ \\
\hline 1 & $S O(n)$ & $n$ & 1 & $(n, n)$ \\
2 & $S O(p) \times S O(n+1-p)$ & $n$ & 2 & $(p, n-p)$ \\
3 & $S O(3)$ & 3 & 3 & $(1,1)$ \\
4 & $S U(3)$ & 6 & 3 & $(2,2)$ \\
5 & $S p(3)$ & 12 & 3 & $(4,4)$ \\
6 & $F_{4}$ & 24 & 3 & $(8,8)$ \\
7 & $S O(5)$ & 8 & 4 & $(2,2)$ \\
8 & $U(5)$ & 18 & 4 & $(4,5)$ \\
9 & $S O(m) \times S O(2), m \geq 3$ & $2 m-2$ & 4 & $(1, m-2)$ \\
10 & $S(U(m) \times U(2)), m \geq 2$ & $4 m-2$ & 4 & $(2,2 m-3)$ \\
11 & $S p(m) \times S p(2), m \geq 2$ & $8 m-2$ & 4 & $(4,4 m-5)$ \\
12 & $(\operatorname{Spin}(10) \times S O(2)) / \mathbb{Z}_{4}$ & 30 & 4 & $(6,9)$ \\
13 & $S O(4)$ & 6 & 6 & $(1,1)$ \\
14 & $G_{2}$ & 12 & 6 & $(2,2)$ \\
\hline
\end{tabular}

The metric $h$ defines a norm $\|\cdot\|_{h}$ on $\mathfrak{g}=\operatorname{Lie}(G)$ such that

$$
\|W\|_{h}=\max _{x \in S^{n}(1)}|W(x)|_{h}
$$

for each $W \in \mathfrak{g}=\operatorname{Lie}(G)$ viewed as Killing vector field of $S^{n}(1)$. Viewing $W$ as a matrix in so $(n+1)$ instead, then we have

$$
\begin{aligned}
\|W\|_{h} & =\max \left\{\langle W x, W x\rangle^{1 / 2} \text { for all } x \in S^{n}(1) \subset \mathbb{R}^{n+1}\right\} \\
& =\max \{c \mid \pm c \sqrt{-1} \text { are eigenvalues of } W\},
\end{aligned}
$$

where $\langle\cdot, \cdot\rangle$ is the standard Euclidean inner product.

Theorem 4.5 indicates that, if we choose the Killing vector field $W$ from the open $\|\cdot\|_{h}$-unit ball in $\mathfrak{g}$, i.e. $\|W\|_{h}<1, M$ is tangent to $W$ and remains isoparametric after the navigation.

The subtle issue here is that $M$ may not be $K$-homogeneous, i.e. a homogeneous hypersurface of $\left(S^{n}, F\right)$. The following lemma indicates any $K$-homogeneous hypersurface in $\left(S^{n}, F\right)$ are tangent to $W$, i.e. an isoparametric hypersurface indicated in Theorem 4.5.

Lemma 5.1 If $M$ is $K$-homogeneous, then it is tangent to the vector field $W$ in the navigation datum.

Proof When $W$ is viewed as a matrix in $\mathfrak{g} \subset \operatorname{so}(n+1)$, it commutes with $\mathfrak{k}=\operatorname{Lie}(K)$. We only need to prove $W \in \mathfrak{k}$. Assume conversely that $W \notin \mathfrak{k}$, then the closure $K^{\prime}$ in $G$ for the group generated by the Lie subalgebra $\mathbb{R} W \oplus \mathfrak{k}$ (which is a closed subgroup of $G$ ) acts transitively on $S^{n}$. The semi-simple part of $\mathfrak{k}^{\prime}=\operatorname{Lie}\left(K^{\prime}\right)$ coincides with that of $\mathfrak{k}$. According to the classification of effective transitive group actions on spheres $[4,19]$, the semi-simple part of $\mathfrak{k}$ must be $\operatorname{su}(m)$ when $n+1=2 m$ is an even number, or $\operatorname{sp}\left(m^{\prime}\right)$ when $n+1=4 m^{\prime}$ can be divided by 4 . In either case, $K$ acts transitively on $S^{n}$, which cannot preserve $M$. This is a contradiction which ends the proof of the lemma.

The remaining task of this section is to classify all $K$-homogeneous hypersurfaces, i.e. all homogeneous isoparametric hypersurfaces of $\left(S^{n}, F\right)$. 
Assume $M \subset S^{n}$ is a $K$-homogeneous hypersurface. By a suitable orthogonalconjugation, we can identify the Killing vector field $W$ with the matrix

$$
W=\operatorname{diag}\left(0_{n_{0}}, \lambda_{1} J_{2 n_{1}}, \ldots, \lambda_{k} J_{2 n_{k}}\right),
$$

where $0<\lambda_{1}<\lambda_{2}<\cdots<\lambda_{k}<1,0_{n_{0}}$ is the $n_{0} \times n_{0}$-zero matrix, and $J_{2 n_{i}}$ is the skew symmetric $2 n_{i} \times 2 n_{i}$-matrix $J_{2 n_{i}}=\left(\begin{array}{ll}0 & I_{n_{i}} \\ -I_{n_{i}} & 0\end{array}\right), n_{i}>0$ when $i>0$, and $n_{0}$ can be 0 .

When $n_{0}>0$ and $k=2$, or $k>2$, the action of $I_{o}\left(S^{n}, F\right)$ on $S^{n}$ has a cohomogeneity bigger than 1 . So in this case, $M$ can not be $K$-homogeneous.

When $n_{0}=0$ and $k=2$, or $n_{0}>1$ and $k=1$, or $n_{0}=1$ and $k=1, I_{o}\left(S^{n}, F\right)=$ $U\left(m_{1}\right) \times U\left(m_{2}\right)$ with $2 m_{1}+2 m_{2}=n+1$, or $S O\left(n_{0}\right) \times U\left(m_{1}\right)$ with $n_{0}+2 m_{1}=n+1$, or $U(m)$ with $2 m+1=n$, respectively. In either case, $W$ is in the centre of $\operatorname{Lie}\left(I_{o}\left(S^{n}, F\right)\right)$, and $K=I_{o}\left(S^{n}, F\right)$. The action of $I_{o}\left(S^{n}, F\right)$ on $S^{n}$ is of cohomogeneity one, so the $K$ homogeneous $M$ must be an orbit of it, which is an isoparametric hypersurface in $S^{n}(1)$ with 1 or 2 distinct principal curvatures.

When $n_{0}=0$ and $k=1, I_{o}\left(S^{n}, F\right)=U(m)$ with $2 m=n+1$ acts transitively on $S^{n}(1)$. In this case, $W$ is a Killing vector field of constant length (or $C K$-vector field in short) on $S^{n}(1)$. Notice $K$ is the connected centralizer of $W$ in $G$. The assumption that $M$ is $K$ homogeneous implies that if we present $M$ as the $G$-homogeneous space $M=G / H$ with $\mathfrak{h}=\operatorname{Lie}(H)$, then

$$
\mathfrak{g}=\mathfrak{h}+\mathfrak{k} .
$$

This equality has been studied by Onishchick [23]. A more geometric way to interpret it, which will be applied in later discussions, is that the restriction of $W$ to $M$ defines a nonzero Killing vector field of constant length (or CK-vector field in short) on $G / H$, with respect to all $G$ homogeneous metrics. In particular, we can choose the Riemannian $G$-normal homogeneous metric for $G / H$.

This observation is valid for all hypersurfaces and focal manifolds in the isoparametric foliation associated to $M$. Assume $M$ is a regular level set for the isoparametric function $f: S^{n}(1) \rightarrow[-1,1]$ such that $f^{-1}( \pm 1)$ are the two focal submanifold. Then, for each $t \in[-1,1], K$ preserves and acts transitively on the level set $f^{-1}(t)$. If we present $f^{-1}(t)=$ $G / H_{t}$ with Lie $\left(H_{t}\right)=\mathfrak{h}_{t}$, then the restriction of $W$ to each $f^{-1}(t)$ defines a CK-vector field on $G / H_{t}$, with respect to all $G$-homogeneous metrics, the Riemannian $G$-normal homogeneous ones.

We will use the following two results for CK-vector fields on Riemannian normal homogeneous spaces.

Theorem 5.2 Let $G$ be a compact connected simple Lie group and $H$ a closed subgroup with $0<\operatorname{dim} H<\operatorname{dim} G$. Fix a Riemannian normal metric on $G / H$. Suppose that there is a nonzero vector $v \in \mathfrak{g}$ defining a $C K$-vector field on $G / H$. Then, up to a finite cover, the only possibilities of $G / H$ are $S^{2 n-1}=S O(2 n) / S O(2 n-1), S^{7}=\operatorname{Spin}(7) / G_{2}$ and $S U(2 n) / S p(n)$.

Proposition 5.3 Assume $\mathrm{G} / \mathrm{H}$ is an irreducible Riemannian normal homogeneous space with $G$ compact and semi-simple. Denote

$$
\mathfrak{g}=\mathfrak{g}_{1} \oplus \cdots \oplus \mathfrak{g}_{r}
$$

the direct sum decomposition of $\mathfrak{g}=\operatorname{Lie}(G)$, where each $\mathfrak{g}_{i}$ is simple. Denote $\pi_{i}$ the projection from $\mathfrak{g}$ to $\mathfrak{g}_{i}$ according to this decomposition. Assume for each $i$, the $\pi_{i}$-image of $\mathfrak{h}=\operatorname{Lie}(H)$ has a dimension strictly between 0 and $\operatorname{dim} \mathfrak{g}_{i}$, and $\xi=\xi_{1}+\cdots+\xi_{r} \in \mathfrak{g}$ defines a 
$C K$-vector field on the normal homogeneous space $G / H$. Then, for each $i$, $\xi_{i}$ defines a $C K$ vector field on the Riemannian normal homogeneous space $G_{i} / H_{i}$ where $\operatorname{Lie}\left(G_{i}\right)=\mathfrak{g}_{i}$ and $\operatorname{Lie}\left(H_{i}\right)=\pi_{i}(\mathfrak{h})$.

Theorem 5.2 is a restatement of Theorem 1.1 in [36], and Proposition 5.3 is part of Theorem 7.6 in [34] for only the Riemannian case.

In the following, we discuss case by case for all fourteen possible choices of $G=$ $I_{o}\left(S^{n}, M, h\right)$ in the table above.

In Case 1 with $G=S O(n)$, the focal manifolds are zero dimensional, which do not admit nonzero CK-vector fields.

In Case 2 with $G=S O(p) \times S O(n+1-p)$, the focal manifolds are two unit spheres. So both $p$ and $n+1-p$ must be even, i.e. both focal manifolds are odd-dimensional spheres, so that they can admit nonzero CK-vector fields. In this case $K=U\left(m_{1}\right) \times U\left(m_{2}\right)$ with $2 m_{1}+2 m_{2}=n+1$ and the CK-vector field $W$ is chosen from the centre of $\operatorname{Lie}\left(I_{o}\left(S^{n}, F\right)\right)=$ $u\left(m_{1}+m_{2}\right)$.

For the cases from Case 3 to Case 7, and Case 14, the group $G$ is simple and $W \in \mathfrak{g}$ defines a nonzero CK-vector field on the Riemannian normal homogeneous spaces $G / H_{ \pm 1}$ (they are the focal submanifolds, but not endowed with the submanifold metric). It is a contradiction because they are not the ones listed in Theorem 5.2.

In Case $13, G=S O(4)$ has the same dimension as $M$. So its proper subgroup $K$ cannot act transitively on $M$.

In Case 11 with $G=S p(m) \times S p(2)$ and $m \geq 2$, one of the focal manifolds is

$$
(S p(m) \times S p(2)) /(\Delta S p(1) \times S p(m-1) \times S p(1)),
$$

where $\Delta S p(1)$ is a diagonal $S p(1)$ in the product $S p(m) \times S p(2)$, and $S p(m-1)$ and the other $S p(1)$ are contained in $S p(m)$ and $S p(2)$, respectively. Theorem 5.3 indicates $W$ defines a nonzero CK-vector field on either $\mathbb{H} \mathrm{P}^{m-1}=S p(m) / S p(m-1) S p(1)$ or $\mathbb{H} \mathrm{P}^{1}=$ $S p(2) / S p(1) S p(1)$. But neither one admits such a CK-vector field by Theorem 5.2.

For all the remaining cases, $G$ contains a one-dimensional centre. If we take $W$ from the centre of $\mathfrak{g}$, then obviously $K=G$ acts transitively on $M$, i.e. $M$ is a homogeneous isoparametric hypersurface in $\left(S^{n}, F\right)$. I will prove that $W$ can only be chosen from the centre. In the following cases, I will assume conversely that $W$ is not in the centre, and prove the claim by contradiction.

In Case 8 with $G=U(5)$, then the maximal possible dimension for $K$ is 17 (which is taken by $K=U(4) \times U(1)$ ), i.e. $K$ cannot act transitively on $M$ which dimension is 18 .

In Case 9 with $G=S O(m) \times S O(2)$ and $m \geq 3$, we denote $M=G / H$ and $H^{\prime}$ is projection image of $H$ in the $S O(m)$-factor. Then $K=K^{\prime} \times S O(2)$, and we can get $\mathfrak{k}^{\prime}+\mathfrak{h}^{\prime}=s o(m)$ from (5.4), in which $\mathfrak{k}^{\prime}=\operatorname{Lie}\left(K^{\prime}\right)$ and $\mathfrak{h}^{\prime}=\operatorname{Lie}\left(H^{\prime}\right)$. So the $s o(m)$-factor $W^{\prime}$ of $W$ defines a nonzero $\mathrm{CK}$-vector field on the Riemannian normal homogeneous space $S O(m) / H^{\prime}$. Notice that $2 m-3 \leq \operatorname{dim} S O(m) / H^{\prime} \leq 2 m-2$. When $m>4$, it is not listed in Theorem 5.2. When $m=3$ or 4, it can be directly checked as in Case 8 that $\operatorname{dim} K<\operatorname{dim} M$, i.e. $K=I_{o}\left(S^{n}, M, F\right)$ can not act transitively on $M$.

In Case 12 with $G=(\operatorname{Spin}(10) \times S O(2)) / \mathbb{Z}_{4}$ and $\operatorname{dim} M=30$, we can apply similar argument as for Case 9 with $m>4$ to get a contradiction.

In Case 10 with $G=S(U(m) \times U(2))$ and $m \geq 2$, one of the focal manifolds corresponds to the $G$-orbit of

$$
\left(\begin{array}{llll}
1 & 0 & \cdots & 0 \\
0 & 0 & \cdots & 0
\end{array}\right) \in \mathbb{C}_{2 \times m},
$$


on which $U(m)$ acts on the columns and $U(2)$ acts on the rows. This focal manifold can be presented as $G / H$, where $H=S(U(1) \times U(1)) \times S(U(m-1) \times U(1))$, in which the first and the third factors belong to $U(m)$ and the other two factors belong to $U(2)$. Denote $\pi, \pi_{1}$ and $\pi_{2}$ the orthogonal projection from $\mathfrak{g}=\operatorname{Lie}(S(U(m) \times U(2)))$ to the direct sum factors $s u(m) \oplus s u(2), s u(m)$ and $s u(2)$ in $\mathfrak{g}$, respectively.

Using the method for Case 12 or for Case 9 with $m>4, W$ defines a nonzero CKvector field on the Riemannian normal homogeneous space $(S U(m) \times S U(2)) / H^{\prime}$ with $\mathfrak{h}^{\prime}=\operatorname{Lie}\left(H^{\prime}\right)=\pi(\mathfrak{h})$. Direct calculation shows that the dimension of $\pi_{1}\left(\mathfrak{h}^{\prime}\right)=\pi(\mathfrak{h})$ $\left(\pi_{2}\left(\mathfrak{h}^{\prime}\right)=\pi(\mathfrak{h})\right.$, respectively) is strictly between 0 and $\operatorname{dim} S U(m)$ (dim $S U(2)$, respectively). By Proposition 5.3, $W$ defines a nonzero CK-vector field for the Riemannian normal homogeneous metric on either $S U(m) / H_{1}$ with $\operatorname{Lie}\left(H_{1}\right)=\pi_{1}\left(\mathfrak{h}^{\prime}\right)$ or $S U(2) / H_{2}$ with $\operatorname{Lie}\left(H_{2}\right)=\pi_{2}\left(\mathfrak{h}^{\prime}\right)$. But they are not listed in Theorem 5.2.

Using Theorem 4.5 and summarizing all the above cases, we have proved the following theorem.

Theorem 5.4 Any homogeneous hypersurface $M$ of a unit sphere $S^{n}(1)=\left(S^{n}, h\right)$ is isoparametric for the Randers sphere $\left(S^{n}, F\right)$ of constant flag curvature, with the navigation datum $(h, W)$ in which $W$ is taken from the open $\|\cdot\|_{h}$-unit ball in the Lie algebra of $G=I_{o}\left(S^{n}, M, h\right)$.

Furthermore, when $\left(S^{n}, F\right)$ is non-Riemannian, $M$ is $K$-homogeneous with $K=$ $I_{o}\left(S^{n}, M, F\right)$ if and only if one of following cases happens:

(1) $G=S O(n)$ with an even number $n$, and $W$ is $O(n+1)$-conjugate to $\operatorname{diag}\left(0, \lambda J_{n}\right)$ with $0<\lambda<1$.

(2) $G=S O(p) \times S O(n+1-p)$ when $p(n+1-p)$ is an even positive number, and $W \in \operatorname{so}(p) \oplus \operatorname{so}(n+1-p)$ is $O(n+1)$-conjugate to $\operatorname{diag}\left(\lambda_{1} J_{2 n_{1}}, \lambda_{2} J_{2 n_{2}}\right)$ with $0<\lambda_{1} \leq \lambda_{2}<1$ or $\operatorname{diag}\left(0_{n_{0}}, \lambda J_{2 n_{1}}\right)$ with $0<\lambda<1$.

(3) $G$ has a one-dimensional centre, i.e.

$$
\begin{aligned}
U(5) \text { with } n & =19, \\
S O(m) \times S O(2) \text { with } m \geq 3 \text { and } n & =2 m-1, \\
S(U(m) \times U(2)) \text { with } m \geq 2 \text { and } n & =4 m-1, \\
(\operatorname{Spin}(10) \times S O(2)) / \mathbb{Z}_{4} \text { when } n & =31 .
\end{aligned}
$$

and $W$ is chosen from $\mathfrak{c}(\mathfrak{g})$ which must be of the form $\lambda J_{n+1} \in \operatorname{so}(n+1)$ with $0<\lambda<1$ up to $O(n+1)$-conjugation.

By Lemma 5.1, Theorem 5.4 and the argument in the proof of Theorem 4.5, we get the following immediate corollary.

Corollary 5.5 If $M$ is a homogeneous hypersurface of a non-Riemannian Randers sphere of constant flag curvature, then it must have one, two or four distinct principal curvatures.

Here the principal curvature is the one with respect to the localization metric $g_{\nabla f}^{F}$ in some neighbourhood of $M$.

\section{Isoparametric hypersurface of the OT-FKM type}

Let $\left\{P_{0}, \ldots, P_{m}\right\}$ with $m \geq 1$ be a symmetric Clifford system on $\mathbb{R}^{2 l}$ (with the standard Euclidean inner product $\langle\cdot, \cdot\rangle$ and the norm $|x|=\langle x, x\rangle^{1 / 2}$ ), i.e. $P_{i}$ 's are real symmetric 
$2 l \times 2 l$-matrices satisfying $P_{i} P_{j}+P_{j} P_{i}=2 \delta_{i j} I_{2 l}$ for all $i$ and $j$. Then, for $x \in \mathbb{R}^{2 l}$ with $|x|=1$,

$$
f(x)=|x|^{4}-2 \sum_{i=0}^{m}\left\langle P_{i} x, x\right\rangle^{2}
$$

defines an isoparametric function on the unit sphere $S^{2 l-1}(1)$, when both $m_{1}=m$ and $m_{2}=l-m-1$ are positive [14]. We call this $f$ (or its regular level sets) an isoparametric function (or isoparametric hypersurfaces, respectively) of the OT-FKM type. An isoparametric hypersurface of the OT-FKM type has four distinct principal curvatures, with multiplicities $m_{1}$ and $m_{2}$ given above.

An isoparametric function $f$ of the OT-FKM type is determined by the Clifford sphere $\Sigma=\Sigma\left(P_{0}, \ldots, P_{m}\right)$ defined as

$$
\Sigma\left(P_{0}, \ldots, P_{m}\right)=\left\{P=\sum a_{i} P_{i} \text { with each } a_{i} \in \mathbb{R} \text { and } \sum a_{i}^{2}=1\right\}
$$

rather than the particular choices for $P_{i}$ 's. Notice that each $P \in \Sigma\left(P_{0}, \ldots, P_{m}\right)$ satisfies $P^{2}=I_{2 l}$ and its eigenspaces $E_{ \pm}(P)$ for \pm 1 have the same dimension $l$.

Conversely, if we assume $m=m_{1} \leq m_{2}=l-m-1$, by Theorem 4.6 in [14], the sphere $\Sigma$ can also be determined by $f$, which was argued as following. The corresponding focal manifold $M_{-}$for the minimal value -1 of $f$ is an $l$-1-dimensional sphere bundle over $\Sigma$. The fibre over each $P \in \Sigma$ is the unit sphere in $E_{+}(P)$. For each $y \in M_{-}$, there exists a unique $P \in \Sigma$ satisfying $P y=y$. By Theorem 4.6 in [14], when $m_{1} \leq m_{2}$,

$$
E_{+}(P)=\mathbb{R} y \oplus \operatorname{span}\left\{\bigcup_{N \in \perp_{y} M_{-} \backslash 0} \operatorname{ker} S_{N}\right\},
$$

where $\perp_{y} M_{-}$is the orthogonal complement of $T_{y} M_{-}$in $T_{y} S^{2 l-1}(1)$, and $S_{N}$ is the shape operator of $M_{-}$at $y$, with respect to the normal vector $N$. Obviously, $E_{+}(P)$ and then $P$ are totally determined. So $\Sigma$ is determined by all points of $M_{-}$and the geometry of $M_{-}$in $S^{2 l-1}(1)$.

For each isoparametric function $f$ of the OT-FKM type, defined by the Clifford sphere $\Sigma$. There are many Killing vector field of the unit sphere $S^{2 l-1}(1)=\left(S^{2 l-1}, h\right)$ which generate flows preserving $f$. They can be used in the navigation datum $(h, W)$ for Theorem 4.5. To determine all the choices for $W$, we only need to determine the Lie algebra they span, in which the set of all possible $W$ 's is the open unit $\|\cdot\|_{h}$-disc.

Firstly, the connected isometry group $S O(m+1)$ of $\Sigma$ can be lifted to a subgroup of $G$. Let $P$ and $Q$ be an orthogonal pair in $\Sigma$, i.e. $P$ and $Q$ in $\Sigma$ satisfy $\langle P, Q\rangle=\frac{1}{2 l} \operatorname{Tr} P Q=0$, or equivalently $P Q+Q P=0$. Their product $P Q$ is a skew symmetric matrix, which defined a Killing vector field $W$ of $S^{2 l-1}(1)$. The conjugations $\operatorname{Ad}\left(e^{t X}\right)$ preserve $\Sigma$, rotating the plane spanned by $P$ and $Q$ and fixing their common orthogonal complement. So the flow generated by $W$ preserves $f$. All such $W$ spanned a Lie algebra $\mathfrak{g}^{\prime}=s o(m+1)$ in $\mathfrak{g}$ with the Spin action on $\mathbb{R}^{2 l}$.

Secondly, there is a subalgebra $\mathfrak{c}(\Sigma)$ of $\mathfrak{g}$, spanned by all $X$ 's in $s o(2 l)$ commuting with each $P \in \Sigma$. This subalgebra can be determined case by case as following.

When $m$ is not a multiple of 4 , up to equivalence, the symmetric Clifford system $\left\{P_{0}, \ldots, P_{m}\right\}$ on $\mathbb{R}^{2 l}$, which is a real representation for the symmetric Clifford algebra $\mathrm{Cl}^{m+1}$, can be decomposed as $k$ copies of the unique irreducible one $\left\{P_{0}^{\prime}, \ldots, P_{m}^{\prime}\right\}$ on $\mathbb{R}^{2 \delta_{m}}$, with $P_{i}=P_{i}^{\prime} \otimes I_{k}$ for each $i$. Denote $\mathbf{A}$ the subalgebra generated by the Clifford system $\left\{P_{0}^{\prime}, \ldots, P_{m}^{\prime}\right\}$ in the matrix algebra over $\mathbb{R}^{2 \delta_{m}}$. It is a simple algebra of the form $\mathbb{R}(\cdot)$, 
$\mathbb{C}(\cdot)$ or $\mathbb{H}(\cdot)$, i.e. the matrix algebras with real, complex, quaternionic coefficients. A matrix $X \in \operatorname{so}(2 l)$ belongs to $\mathfrak{c}(\Sigma)$ if and only if it commutes with all the matrices in $\mathbf{A} \otimes I_{k}$. We can use Schur's Lemma to discuss each of the following cases.

Case 1 When $m=8 q+r$ with $r=1$ or $7, \mathbf{A}=\mathbb{R}\left(2 \delta_{m}\right)$ with $\delta_{m}=2^{4 q}$ when $r=1$ and $\delta_{m}=2^{4 q+3}$ when $r=7$. In this case, $X \in \mathfrak{c}(\Sigma)$ if and only if it can be presented as $X=I_{2 \delta_{m}} \otimes X^{\prime}$ where $X^{\prime}$ is skew symmetric. So $\mathfrak{c}(\Sigma)$ is isomorphic to $s o(k)$.

Case 2 When $m=8 q+r$ with $r=2$ or $6, \mathbf{A}=\mathbb{C}\left(\delta_{m}\right) \subset \mathbb{R}\left(2 \delta_{m}\right)$ with $\delta_{m}=2^{4 q+1}$ when $r=2$ and $\delta_{m}=2^{4 q+3}$ when $r=6$. In this case, $X \in \mathfrak{c}(\Sigma)$ if and only if it can be presented as $X=I_{\delta_{m}} \otimes X^{\prime}$ where $X^{\prime} \in \mathbb{C}(k) \subset \mathbb{R}(2 k)$ is real skew symmetric, i.e. $X^{\prime} \in u(k)$. So $\mathfrak{c}(\Sigma)$ is isomorphic to $u(k)$.

Case 3 When $m=8 q+r$ with $r=3$ or $5, \mathbf{A}=\mathbb{H}\left(\delta_{m} / 2\right) \subset \mathbb{R}\left(2 \delta_{m}\right)$ with $\delta_{m}=2^{4 q+2}$ when $r=3$ and $\delta_{m}=2^{4 q+3}$ when $r=5$. In this case, $X \in \mathfrak{c}(\Sigma)$ if and only if it can be presented as $X=I_{\delta_{m} / 2} \otimes X^{\prime}$ where $X^{\prime} \in \mathbb{H}(k) \subset \mathbb{R}(4 k)$ is real skew symmetric, i.e. $X^{\prime} \in \operatorname{sp}(k)$. So $\mathfrak{c}(\Sigma)$ is isomorphic to $s p(k)$.

When $m$ is a multiple of 4 , there exist exactly two distinct irreducible Clifford systems $\left\{P_{0}^{\prime}, \ldots, P_{m}^{\prime}\right\}$. They are on real vector spaces of the same dimension $2 \delta_{m}$, which will be denoted as $\mathbf{V}_{1}$ and $\mathbf{V}_{2}$, respectively. Denote $\mathbf{A}_{i}, i \in\{1,2\}$, the subalgebra generated by each irreducible Clifford system $\left\{P_{0}^{\prime}, \ldots, P_{m}^{\prime}\right\}$ in the matrix algebra over $\mathbf{V}_{i}$.

The Clifford system $\left\{P_{0}, \ldots, P_{m}\right\}$ can be regarded as the sum of $k_{1}$ copies of the irreducible one on $\mathbf{V}_{1}$ and $k_{2}$ copies of that on $\mathbf{V}_{2}$, where $k_{1}+k_{2}=k$ and $l=k \delta_{m}$. Up to congruence, we may assume $k_{1} \geq k_{2}$.

Case 4 When $m=8 q+r$ with $r=4$, for each $\mathbf{V}_{i}, \mathbf{A}_{i}=\mathbb{H}\left(\delta_{m} / 2\right)$ with $\delta_{m}=2^{4 q+2}$. In this case, $X \in \mathfrak{c}(\Sigma)$ if and only if $X$ can be presented as $X=I_{\delta_{m} / 2} \otimes\left(X_{1} \oplus X_{2}\right)$, where for each $i, X_{i} \in \mathbb{H}\left(k_{i}\right) \subset \mathbb{R}\left(4 k_{i}\right)$ is real skew symmetric, i.e. $X_{i} \in \operatorname{sp}\left(k_{i}\right)$. So $\mathfrak{c}(\Sigma)$ is isomorphic to $\operatorname{sp}\left(k_{1}\right) \oplus \operatorname{sp}\left(k_{2}\right)$.

Case 5 When $m=8 q$, for each $\mathbf{V}_{i}, \mathbf{A}_{i}=\mathbb{R}\left(2 \delta_{m}\right)$ with $\delta_{m}=2^{4 q-1}$. In this case, $X \in \mathfrak{c}(\Sigma)$ if and only if $X$ can be presented as $X=I_{2 \delta_{m}} \otimes\left(X_{1} \oplus X_{2}\right)$ where $X_{i} \in \operatorname{so}\left(k_{i}\right)$ for each $i$. So $\mathfrak{c}(\Sigma)$ is isomorphic to $s o\left(k_{1}\right) \oplus \operatorname{so}\left(k_{2}\right)$.

Finally, we determine the isomorphic type of $\mathfrak{g}=\operatorname{Lie}\left(I_{o}\left(S^{2 l-1}, M, h\right)\right)$ when $m=m_{1} \leq$ $m_{2}=l-m-1$. We have observed that any $\varphi$ in $I_{o}\left(S^{2 l-1}(1), M, h\right)$ corresponds to an orthogonal matrix $T \in S O(2 l)$, such that the conjugation $\operatorname{Ad}(T)$ preserves the Clifford sphere $\Sigma=\Sigma\left(P_{0}, P_{1}, \cdots, P_{m}\right)$. With the metric defined by $\langle P, Q\rangle=\frac{1}{2 l} \operatorname{Tr} P Q, \Sigma$ is identified with a unit sphere, and $\operatorname{Ad}(T)$ defines an isometry $\tilde{\varphi}$ on $\Sigma$. The group morphism from $\varphi$ to $\tilde{\varphi}$ is surjective, and $\varphi$ belongs to the kernel if and only if the corresponding $T$ commutes with each $P \in \Sigma$. At the Lie algebra level, we have the exact sequence

$$
0 \rightarrow \mathfrak{c}(\Sigma) \rightarrow \mathfrak{g} \rightarrow \operatorname{so}(m+1) \rightarrow 0
$$

and the subalgebra $\mathfrak{g}^{\prime} \subset \mathfrak{g}$ (isomorphic to $s o(m+1)$ ) defined above is a section for this exact sequence. So $\mathfrak{g}$ has the same isomorphic type as $\operatorname{so}(m+1) \oplus \mathfrak{c}(\Sigma)$.

To summarize, we have the following theorem.

Theorem 6.1 Let $M$ be an isoparametric hypersurface in the unit sphere $S^{2 l-1}(1)=$ $\left(S^{2 l-1}, h\right)$ of the OT-FKM type, defined by the Clifford system $\left\{P_{0}, \ldots, P_{m}\right\}$, and satisfy $m=m_{1} \leq m_{2}=l-m-1=k \delta_{m}-m-1$. Then, $M$ is isoparametric for the Randers sphere $\left(S^{2 l-1}, F\right)$ when the Killing vector field $W$ in the navigation datum $(W, h)$ is chosen from the open $\|\cdot\|_{h}$-unit ball in Lie algebra of $I_{o}\left(S^{2 l-1}, M, h\right)$ which is isomorphic to one of the following: 
(1) $\operatorname{so}(m+1) \oplus \operatorname{so}(k)$ when $m \equiv 1$ or $7(\bmod 8)$,

(2) $\operatorname{so}(m+1) \oplus u(k)$ when $m \equiv 2 \operatorname{or} 6(\bmod 8)$,

(3) $s o(m+1) \oplus s p(k)$ when $m \equiv 3$ or $5(\bmod 8)$,

(4) $s o(m+1) \oplus s p\left(k_{1}\right) \oplus s p\left(k_{2}\right)$ with $k_{1}+k_{2}=k$ when $m \equiv 4(\bmod 8)$,

(5) $\operatorname{so}(m+1) \oplus \operatorname{so}\left(k_{1}\right) \oplus \operatorname{so}\left(k_{2}\right)$ with $k_{1}+k_{2}=k$, when $m \equiv 0(\bmod 8)$.

Almost all isoparametric hypersurfaces of the OT-FKM type satisfy the condition $m_{1}=$ $m \leq l-m-1=m_{2}$. Even when $m_{1}>m_{2}$, we can still find a subalgebra of $\mathfrak{g}$ with the same isomorphism type as listed in Theorem 6.1, from which we can choose $W$ for the navigation and get a special isoparametric hypersurface of $\left(S^{n}, F\right)$.

If an isoparametric hypersurface $M \subset S^{n}(1)$ of the OT-FKM type satisfying $m_{1}>m_{2}$ and $\left(m_{1}, m_{2}\right) \neq(8,7)$, either it is homogeneous, or it is congruent to another isoparametric hypersurface of the OT-FKM type with $m_{1}$ an $m_{2}$ switched. In either case, we can determine all the possible choices of $W$, i.e. classify the Randers metrics $F$ for the special isoparametric hypersurfaces in Theorem 4.5.

Acknowledgements I would like to thank College of Mathematics and System Science, Xinjiang University, for its hospitality during the preparation of this paper. I would also like to thank Zhongmin Shen, Qun He, Chao Qian, and Songting Yin for helpful discussions.

\section{References}

1. Bao, D., Chern, S.S., Shen, Z.: An Introduction to Riemann-Finsler Geometry. Springer, New York (2000)

2. Bao, D., Robles, C.: On Randers metrics of constant curvature. Rep. Math. Phys. 51, 9-42 (2003)

3. Bao, D., Robles, C., Shen, Z.: Zermelo navigation on Riemannian manifolds. J. Differ. Geom. 66, 377-435 (2004)

4. Borel, A.: Some remarks about Lie groups transitive on spheres and tori. Bull. AMS 55, 580-587 (1940)

5. Bryant, R.: Finsler structures on the 2 -sphere satisfying $K=1$, Finsler Geometry. Contemp. Math. 196, 27-42 (1996)

6. Cartan, E.: Familles de surfaces isoparamétriques dans les espaces à courbure constante. Ann. Mat. Pura Appl. 17, 177-191 (1938)

7. Cartan, E.: Sur des familles remarquables d'hypersurfaces isoparamétriques dans les espaces sphériques. Math. Z. 45, 335-367 (1939)

8. Cartan, E.: Sur des familles d'hypersurfaces isoparamétriques des espaces sphériques à 5 et à 9 dimensions. Rev. Univ. Tucuman. Ser. A 1, 5-22 (1940)

9. Cecil, T., Chi, Q.S., Jensen, G.: Isoparametric hypersurfaces with four principal curvatures. Ann. Math. 166, 1-76 (2007)

10. Chi, Q.S.: Isoparametric hypersurfaces with four principal curvatures, II. Nagoya Math. J. 204, 1-18 (2011)

11. Chi, Q.S.: Isoparametric hypersurfaces with four principal curvatures, III. J. Differ. Geom. 94, 469-504 (2013)

12. Chi, Q.S.: Isoparametric hypersurfaces with four principal curvatures, IV. arXiv: 1605.00976

13. Dorfmeister, J., Neher, E.: Isoparametric hypersurfaces, case $g=6, m=1$. Commun. Algebra 13, 2299-2368 (1985)

14. Ferus, D., Karcher, H., Münzner, H.F.: Clifford algebras and new isoparametric hypersurfaces. Math. Z. 177, 479-502 (1981)

15. Hsiang, W.Y., Lawson, H.B.: Minimal submanifolds with low cohomogeneity. J. Differ. Geom. 5, 1-38 (1971)

16. He, Q., Yin, S., Shen, Y.: Isoparametric hypersurfaces in Minkowski spaces. Differ. Geom. Appl. 47(3-4), 133-158 (2015)

17. He, Q., Yin, S., Shen, Y.: Isoparametric hypersurfaces in Funk spaces. Sci. China Math. (2016). doi:10. 1007/s11425-000-0000-0

18. Miyaoka, R.: Isoparametric hypersurfaces with $(g, m)=(6,2)$. Ann. Math. 177, 53-110 (2013)

19. Montgomery, D., Samelson, H.: Transformation groups of spheres. Ann. Math. 44, 454-470 (1943)

20. Münzner, H.F.: Isoparametrische hyperflächen in sphären, I. Math. Ann. 251, 57-71 (1980) 
21. Münzner, H.F.: Isoparametrische hyperflächen in sphären, II. Math. Ann. 256, 215-232 (1981)

22. Nomizu, K.: Some results in E. Cartan's theory of isoparametric families of hypersurfaces. Bull. Am. Math. Soc. 79, 1184-1188 (1973)

23. Onishchick, A.L.: Inclusion relations among transitive compact transformation groups, Trudy Moskov. Mat. Obsc. 11, 199-242 (1962); English transl., Am. Math. Soc. Transl. 50(2), 5-58 (1966)

24. Ozeki, H., Takeuchi, M.: On some types of isoparametric hypersurfaces in spheres, I. Tohoku Math. J. 27, 515-559 (1975)

25. Ozeki, H., Takeuchi, M.: On some types of isoparametric hypersurfaces in spheres, II. 28, 7-55 (1976)

26. Alvarez Paiva, J.C., Duran, C.E.: Isometric submersion of Finsler manifolds. Proc. Am. Math. Soc. 129, 2409-2417 (2001)

27. Qian, C., Tang, Z.: Recent progress in isoparametric functions and isoparametric hypersurfaces. In: Suh, Y.J., Berndt, J., Ohnita, Y., Kim, B.K., Lee, H. (eds.) Real and Complex Submanifolds, Daejeon, Korea, August 2014. Springer Proceedings in Mathematics and Statistics Book Series, vol. 106

28. Segre, B.: Familie di ipersuperficie isoparametriche negli spazi euclidei ad un qualunque numero di dimensioni. Atti. Accad. Naz. Lincei Rend. VI. Ser. 27, 203-207 (1938)

29. Shen, Z.: Lectures on Finsler geometry. World Scientific, Singapore (2001)

30. Somigliana, C.: Sulle relazione fra il principio di Huygens e l'ottica geometrica. Atti. Accad. Sci. Torino Cl. Sci. Fis. Mat. Nat. 24, 974-979 (1918-1919)

31. Thorbergsson, G.: A survey on isoparametric hypersurfaces and their generalizations. In: Dillen, F.J.E., Verstraelen, L.C.A. (eds.) Handbook of Differential Geometry, vol. I, pp. 963-995. North-Holland, Amsterdam (2000)

32. Takagi, R., Takahashi, T.: On the principal curvature of homogeneous hypersurfaces in a sphere, Diff. Geom. in Honor of Kentaro Yano (1972) 469-481

33. Wang, Q.M.: Isoparametric functions on Riemannian manifolds I. Math. Ann. 277, 639-646 (1987)

34. Wolf, J.A., Podestà, F., Xu, M.: Toward a classification of Killing vector fields of constant length on pseudo-Riemannian normal homogeneous spaces. J. Differ. Geom. 109, 519-532 (2017)

35. Xing, H.: The geometric meaning of Randers metrics with isotropy S-curvature. Adv. Math. 34(6), $717-$ $730(2005)$

36. Xu, M., Wolf, J.A.: Killing vector fields of constant length on Riemannian normal homogeneous spaces. Transform. Groups 21(3), 871-902 (2016) 